http://www.jfas.info

\title{
STEAM TEMPERATURE CONTROL OF ESSENTIAL OIL EXTRACTION SYSTEM USING FUZZY-FOPI CONTROLLER
}

\author{
M. Tajjudin", N. Ishak, M. H. F. Rahiman and R. Adnan \\ Faculty of Electrical Engineering, Universiti Teknologi MARA, 40450 Shah Alam, Selangor, \\ Malaysia
}

Published online: 05 October 2017

\begin{abstract}
This research proposed a closed-loop temperature control using a self-tuning fuzzy fractional-order PI (FOPI) controller to overcome the problem. The controller will regulate the steam temperature at a desired level to protect the oil from excessive heat. Self-tuning capability of fuzzy rules was found to facilitate the tuning using only information about output error and rate of output error. The proposed controller was found to be more efficient in terms of energy consumption and produce better response compared to self-tuning fuzzy PI. Essential oil quality assessment was also performed for citronella oil samples that were extracted at $85^{\circ} \mathrm{C}$ and $100^{\circ} \mathrm{C}$ (uncontrolled). Significant improvement had been observed in the sample extracted at $85^{\circ} \mathrm{C}$ based on physical and chemical evaluations using refractive index measurement and GC-MS analysis.
\end{abstract}

Keywords: fuzzy control; fractional-order PID; essential oil extraction; hydro-steam distillation.

Author Correspondence, e-mail: mazidah@salam.uitm.edu.my

doi: $\underline{\text { http://dx.doi.org/10.4314/jfas.v9i4s.14 }}$ 


\section{INTRODUCTION}

Essential oils are complex mixtures of volatile substances mainly derived from aromatic plants. They are primarily used in flavour, fragrance, cosmetics and pharmaceutical industries [1]. The properties of essential oil are unique and can be distinguished by the composition of these chemical constituents [2]. They can be affected by external factors including temperature during extraction process [3].

Generally, hydro-steam distillation is the most practical and affordable extraction method especially for small-scale industries. This method is almost similar to hydro distillation except that the botanic materials are supported above the boiling water. Consequently, total capacity of the distillation tank is reduced which is one of the disadvantage of hydro-steam distillation. Major drawback from this method is that the plant material can get wet by the condensed steam and slowing down the distillation process. However, this effect can be minimized by loosely packing the plant material so that the steam can go up more easily.

Theoretically, hydro-steam distillation method produced better quality essential oil compared to hydro distillation but unfortunately, this technique is rarely found in literatures. A comparative study of this method over hydro distillation and steam distillation for lavender oil extraction was conducted by [4], where hydro-steam distillation technique was found to produce higher yield with better oil quality in terms of ester content compared to steam distillation. Furthermore, chemical reactions were minimized in hydro-steam distillation compared to hydro distillation.

Thorough studies regarding the effect of distillation temperature had been established by many researchers in this area. Among all, in [5] who had studied on the effect of temperature over ginger extraction using hydro distillation and steam distillation methods had published significant correlation between temperature and essential oil quality based on the analysis using Differential Scanning Calorimeter (DSC). It suggested that the extraction should be done at lower temperature because ginger oil will burn if heated up to $85^{\circ} \mathrm{C}$ as a sign of decomposition. This finding was supported by the results reported by [6] that compared the Rosa Canina L. essential oils quality, which were extracted at $50^{\circ} \mathrm{C}$ and $100^{\circ} \mathrm{C}$ using dry distillation methods. It was observed that quality of essential oil was decreased with increase in temperature as the content of oxygenated compound was reduced.

In [7] had performed the extraction of Cymbopogon winteranius J. using pressurized liquid carbon dioxide $\left(\mathrm{CO}_{2}\right)$ technique with varying temperature from $8^{\circ} \mathrm{C}$ to $25^{\circ} \mathrm{C}$. The results show that variation in temperature has significant impact on chemical composition of the essential 
oil. Percentage of geraniol increases from $4.49 \%$ to $96.4 \%$ when temperature was set to $8{ }^{\circ} \mathrm{C}$ and $25{ }^{\circ} \mathrm{C}$ respectively. But, content of citronellol and citronellal were not consistent with temperature. However, higher numbers of chemical compounds had been recovered during $20^{\circ} \mathrm{C}$ compared to other temperatures and been selected as the optimal operating temperature in their research.

So far, research had proved that temperature effect can be minimized if the extraction was performed at lower temperature. Hence, this research is aim to introduce low temperature extraction for hydro-steam distillation system. Schematic diagram of the design is shown in Fig. 1.

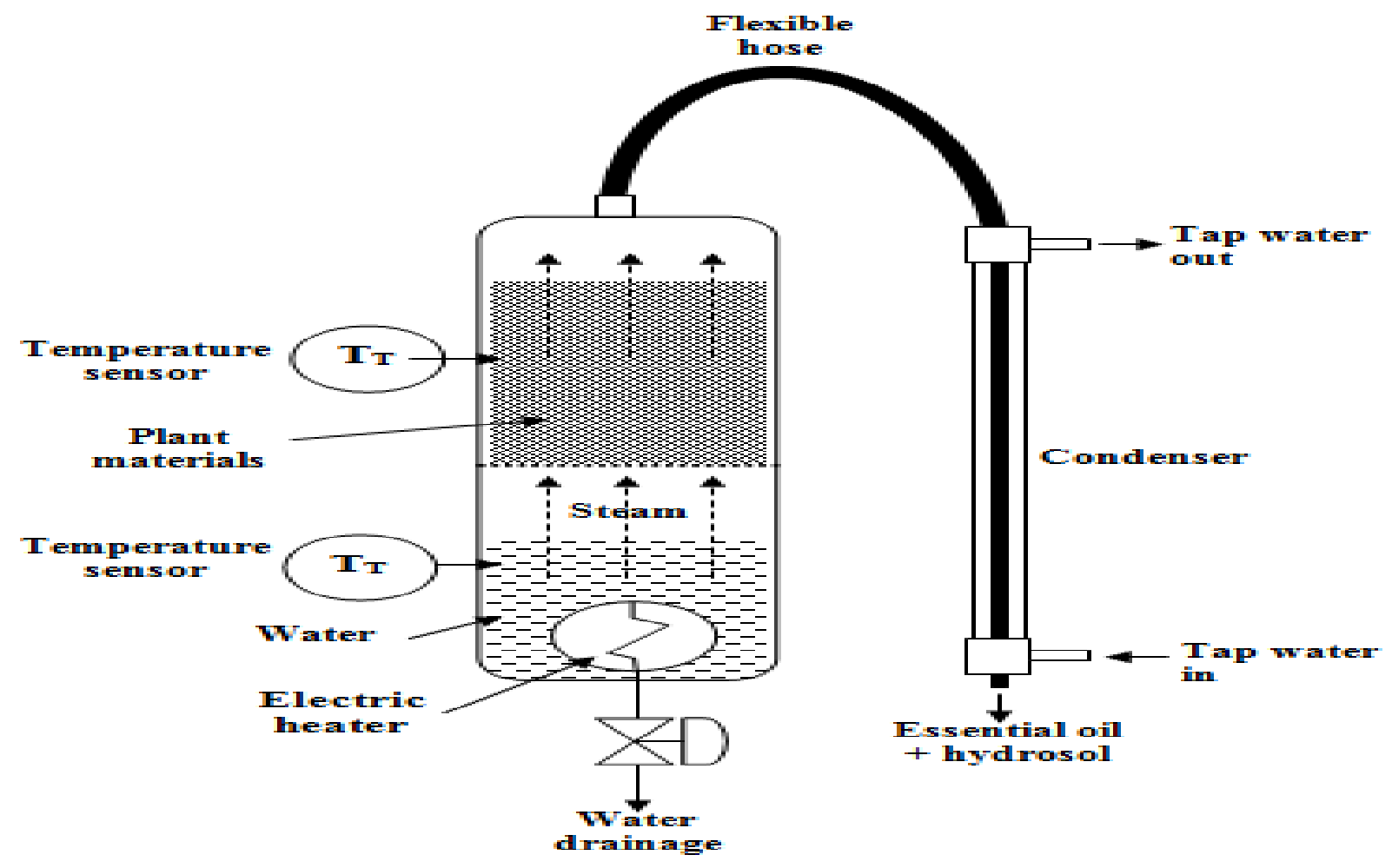

Fig.1. Schematics diagram of hydro-steam distillation plant

Essential oil extraction using hydro-steam distillation technique generates steam inside the distillation tank from boiling water as shown in Fig 1. The steam is released from the bottom and passed through the materials. Along the passage, heat from the steam releases the essential oil from the raw material. Steam with essential oil mixture will pass through the condenser, where it will be condensed into essential oil droplets and hydrosol. The oil can be separated later on using a pipette or any other instruments. A first-order plus dead-time (FOPDT) model of the steam temperature is given in Equation (1).

$G_{1}(s)=\frac{4.5}{(280 s+1)} e^{-25 s}$ 


\section{MATERIAL AND METHOD}

\subsection{Fractional-Order PID Controller}

Fractional-order calculus (FOC) is a generalization of conventional integration and differentiation that include non-integer orders. Fundamental operator representing the fractional-order differential and integration is given in Equation (2), where $\alpha$ is a real number [8].

${ }_{\alpha} D_{t}^{\alpha}= \begin{cases}\frac{d^{\alpha}}{d t^{a}} & , \alpha>0 \\ 1 & , \alpha=0 \\ \int_{a}^{t}(d \tau)^{\alpha} & , \alpha<0\end{cases}$

Linear operator D was interpreted as a differentiator when $\alpha$ is positive and an integrator when $\alpha$ is negative. Otherwise, $\mathrm{D}$ is a unity when $\alpha$ is zero.

The knowledge on fractional calculus was transferred into control engineering by Tustin to control the position of massive object back in 1958. However, the application was halted due to lack of theory and computational limitation of the systems. The agenda was resumed only in early 90's when Oustaloup introduced the idea of fractional-order controller through Commande Robuste d'Ordre Non Entier (CRONE) algorithm which means non-integer robust control in French. The motivation of CRONE design was to achieve iso-damping (constant phase) characteristics that can tolerate the gain and parameter variations. The improvement of the first-generation and second-generation CRONE has been made to allow fractional-order controller of complex number and nonlinear system. It also demonstrated the superior performance of CRONE over the classical PID controller [9].

Later on, in 1999 Podlubny had proposed a generalization of PID, where the integral and differential terms in the PID were presented by non-integer order which was known as fractional-order PID (FOPID) [10]. FOPID introduced an integrator of order $\lambda$ and differentiator of order $\mu$ where $\mathrm{Kc}, \mathrm{Ki}$ and $\mathrm{Kd}$ are the controller's gain. The structure of FOPID is given in Equation (3).

$\frac{U(s)}{E(s)}=K_{c}+\frac{K_{i}}{s^{\lambda}}+K_{d} s^{\mu}$

FOPID is a generalization of the integer PID in such a way that:

- If $\lambda=1$ and $\mu=1$, a classical PID is obtained.

- If $\lambda=1$ and $\mu=0$, a PI controller is obtained.

- If $\lambda=0$ and $\mu=1$, a PD controller is obtained. 
- If $\lambda=0$ and $\mu=0$, a P controller is obtained.

Hence, if $\lambda$ and $\mu$ were set to arbitrary value between 0 and 1 , the controller can be configured to behave within these four possibilities of FOPID control space shown in Fig. 2.

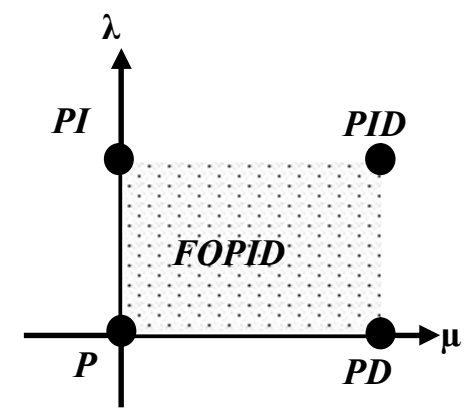

Fig.2. Fractional-order PID control space

An advantage of FOPID over the classical PID is that it provides more degree of freedom for the controller tuning instead of only three in the PID. FOPID allows tuning of the fractional-orders which are $\lambda$ and $\mu$ besides the $\mathrm{Kc}, \mathrm{Ki}$ and $\mathrm{Kd}$.

Since it was introduced, FOPID control had been applied by many researchers, which turned out to produce better performances compared to the classical PID in many applications such as steam temperature control and dc motor and $\mathrm{pH}$ neutralization process respectively [11-12]. Research and applications in fractional-order control (FOC) has gain more interest as the realization of FOC system was becoming easier than before. Many mathematical techniques are used to approximately derive the behaviour of fractional-order differ-integrator from high-order integer approximation. For instance, Oustaloup's approximation make used of recursive distribution of poles and zeros to obtain series of rational functions whose frequency response fit the frequency response of the irrational function within specific frequency band where $\omega_{\mathrm{L}}<\omega<\omega_{\mathrm{H}}$. The method was derived based on approximation of a function given in Equation (4), where $\mu$ is the order and $\mu$ is a positive real number.

$H(s)=s^{\mu}, \quad \mu \in \mathfrak{R}^{+}$

The transfer function of Equation (4) is approximated into a rational transfer function represented by Equation (5) [13].

$s_{\left[\omega_{L}, \omega_{H}\right]}^{\lambda}=k \prod_{n=-N}^{N} \frac{1+s / \omega_{z, n}}{s / \omega_{p, n}}$

where $\mathrm{N}, \omega_{\mathrm{z}, \mathrm{n}}, \omega_{\mathrm{p}, \mathrm{n}}$ and $\mathrm{k}$ are the number of poles and zeros, the location of zero and pole and a constant gain respectively. $\mathrm{N}$ is chosen before the algorithm was run, while the poles and zeros are calculated using the recursive Equation (7) until (9). 
$\omega_{z, 1}=\omega_{l} \sqrt{\eta}$

$\left.\begin{array}{l}\omega_{p, n}=\omega_{z, n} \alpha \\ \omega_{z, n+1}=\omega_{p, n} \eta\end{array}\right\}, n=1 \ldots N$

$\alpha=\left(\frac{\omega_{H}}{\omega_{L}}\right)^{2 / N}$

(8)

$\eta=\left(\frac{\omega_{H}}{\omega_{L}}\right)^{1-(\lambda / N)}$

Fig. 3 shows frequency response obtained using ORA approximation of few fractional differentiators and integrators with $\mathrm{N}=3$. The magnitude curve shows behaviour of a low-pass and high-pass filter for the integrator and differentiator respectively. Magnitude and phase response of each function related to fractional-order, $\lambda$ is given by Equation (10).

$$
\left.\begin{array}{c}
20 \log \left|\hat{s}^{\lambda}\right|_{s=j \omega} \mid=20 \lambda \log (\omega) d B \\
\left.\angle \hat{s}^{\lambda}\right|_{s=j \omega}=\frac{\pi \lambda}{2}
\end{array}\right\} \quad \omega_{l} \leq \omega \leq \omega_{h}
$$

Equation (10) shows that gain and phase is directly dependent on $\lambda$ and can be adjusted between $\pm 20 \mathrm{~dB} / \mathrm{dec}$ and $\pm 90^{\circ}$ by adjusting the fractional order.

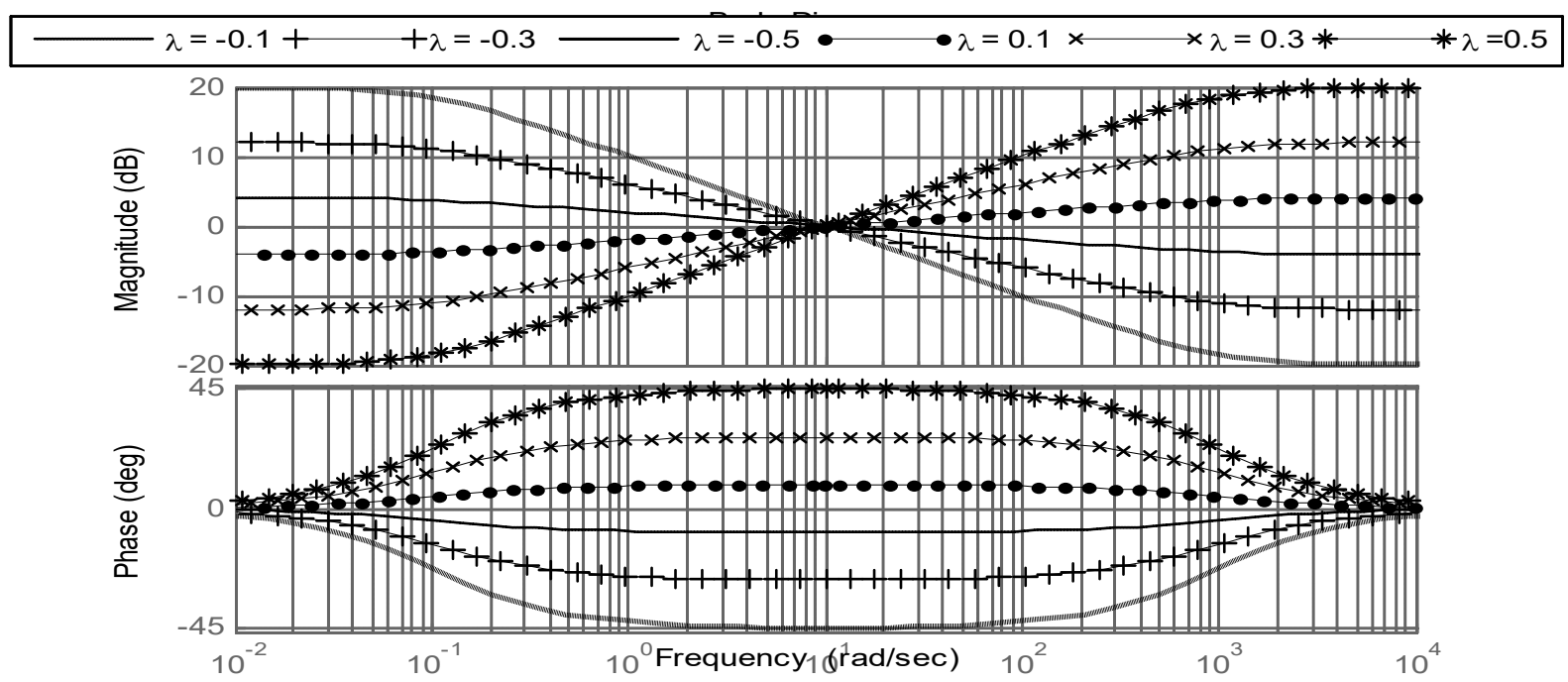

Fig.3. Fractional-order differ-integrators approximation using ORA method with $\mathrm{N}=3$ Unfortunately, ORA approximation always lead to a feedback system that unable to track the reference because it approximates the fractional-order integrator term with no pole at the 
origin. Later on, in [14] proposed a solution by using an auxiliary function given by Equation (11) to minimize the error. This function will be referred to as error filter in this paper.

$G_{f}(s)=\frac{s+n}{s}$

The error filter introduced a pole at the origin to increase the low frequency magnitude and a zero at $\mathrm{n}$ to limit its high frequency cut-off. Hence, the frequency response was fulfilled with better steady-state error as shown in Fig. 4.

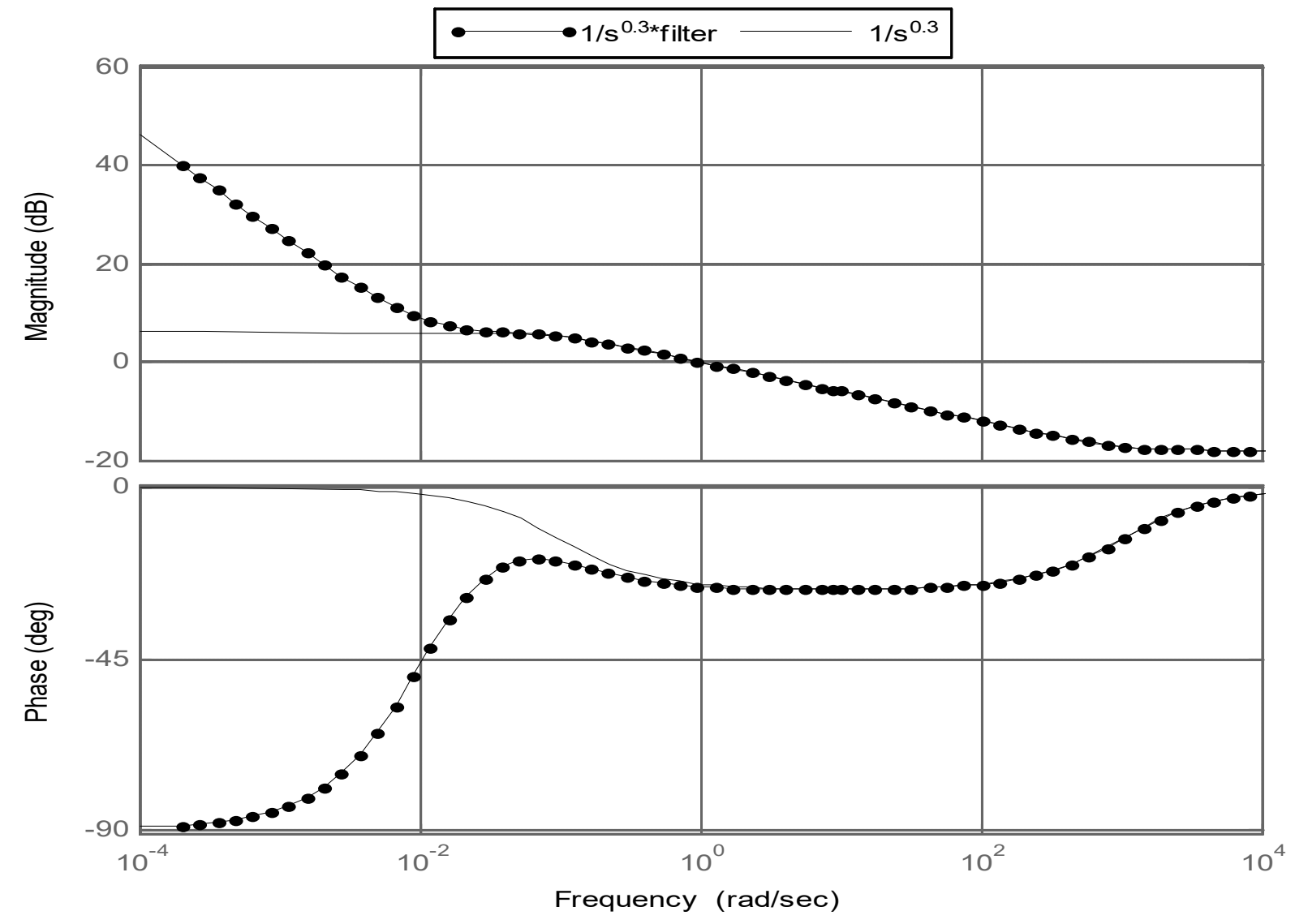

Fig.4. Implementation of $1 / \mathrm{s}^{0.3}$ with error filter

\subsection{Design of Fuzzy FOPI Controller}

Fuzzy logic offers an interesting approach to control complex processes based on intuition and experience of human. The algorithm can be used to evaluate the human linguistic rules and programmed into fuzzy logic language formally known as fuzzy inference. This strategy can be expressed linguistically as a set of imprecise conditional using 'if' and 'then' logical statements to relate significant measured variables to the control actions.

Fuzzy logic control (FLC) has gaining popularity among practicing engineers. It has merit in the sense that no process model was required for the control design. This made fuzzy logic control a preferred alternative when dealing with complex ill-defined processes having 
uncertain dynamics and nonlinearities. In industrial point of view, fuzzy logic technology provides an efficient solution that make used of experimental experience rather than mathematical model-based control design. Recently, in [15] had published the results on the application of fuzzy logic to facilitate the decision making process of sesame crops operations. Other than that, fuzzy logic had been applied in a decision making of a multi-mode driving electric vehicle for energy efficiency [16]. These applications served as evidence that fuzzy logic technology was superior in cases when control operation needs human expertise intervention, especially when dealing with multi-input control that can be implemented more easily without rendering with complex mathematical solutions.

Trend of fuzzy control research is currently looking towards using fuzzy with FOPID control structure for better control performances. The adaptive mechanism provides by fuzzy logic can minimize the trade-off between PID parameters tuning and its fractional-order terms when either term can be selected in an adaptive manner. In [17] had applied self-tuning fuzzy FOPID for speed control of a dc motor using the same approach as fuzzy PID. In their study, initial value of the PID was determined using Ziegler-Nichols while Genetic Algorithm (GA) [28] optimization technique was used to determined optimal value of the fractional-orders. In [18] also applied the same technique where the fuzzy rules were used to update PID [27] gain, while fractional-orders were determined manually and kept constant. Input to the fuzzy inference came from the control error and the rate of control error while 49 fuzzy rules were designed exclusively for each gain. The same approach was used by [19] for trajectory control of robotic manipulator. Recent research had reported to implement fuzzy FOPID on distillation column [20], while others had demonstrated different PID structure with fuzzy FOPID [21-22].

In this research, fuzzy controller was designed using error and rate of error as the input signals and $\mathrm{Kp}$ and $\mathrm{Ki}$ as the output signals. In this research, range of error signal was set between $-70^{\circ} \mathrm{C}$ to $70^{\circ} \mathrm{C}$ to represent full range of steam temperature. The range was then divided into several membership functions and designated with its own linguistic variable. Gaussian membership function was applied as shown in Fig. 5. 


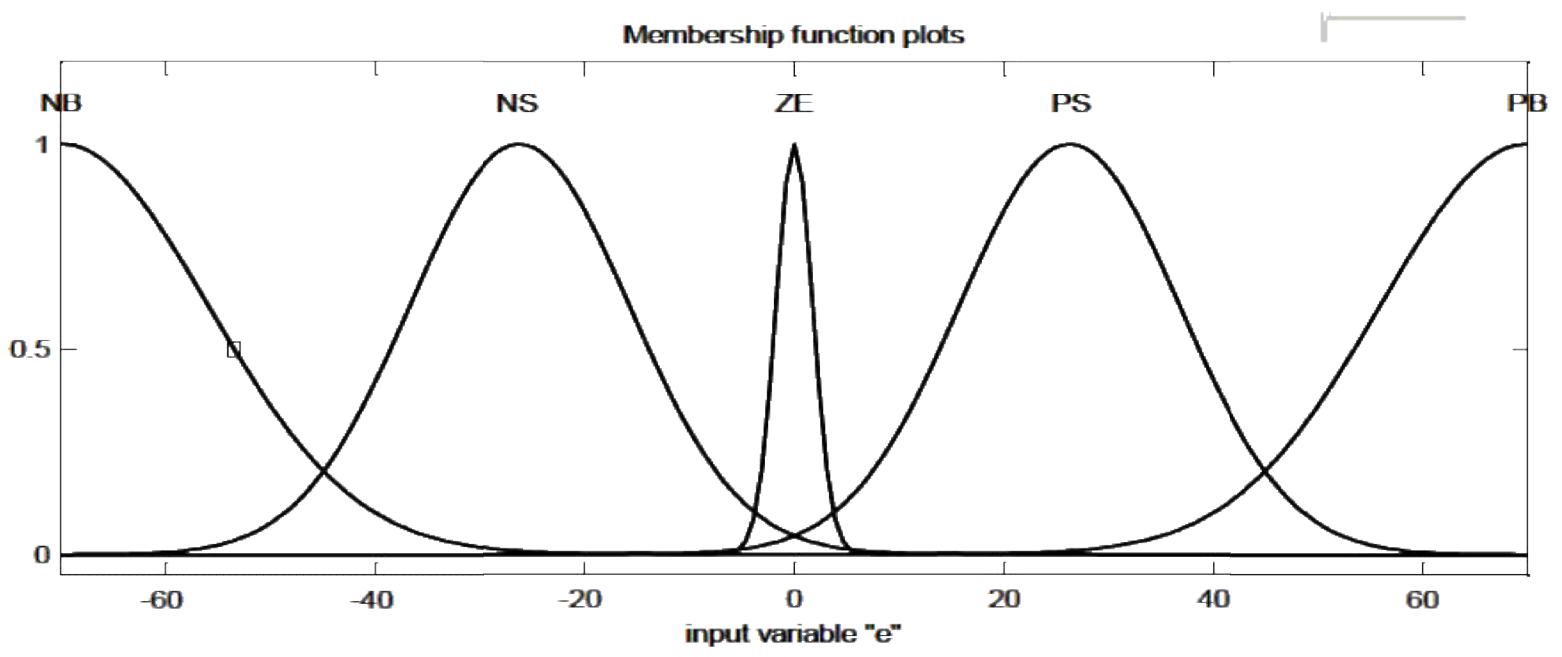

Fig.5. Membership function of error

Linguistic variables for error were defined as Zero (ZE), Positive Big (PB), Positive Small (PS), Negative Big (NB) and Negative Small (NS). Range of Zero (ZE) error was minimized to $\pm 1.75^{\circ} \mathrm{C}$ to prevent excessive control action around the steady-state while small and big error had larger spread which is $\pm 10.25^{\circ} \mathrm{C}$ and $\pm 14^{\circ} \mathrm{C}$ respectively.

Rate of error was designed within small range because steam temperature reading is highly influenced by measurement noise and tend to fluctuates. Small change in rate of error represents by Zero (ZE) was designed to have small variance so that fluctuation in error rate will only cause small changes in control action. Membership function of rate of error was described in Fig. 6.

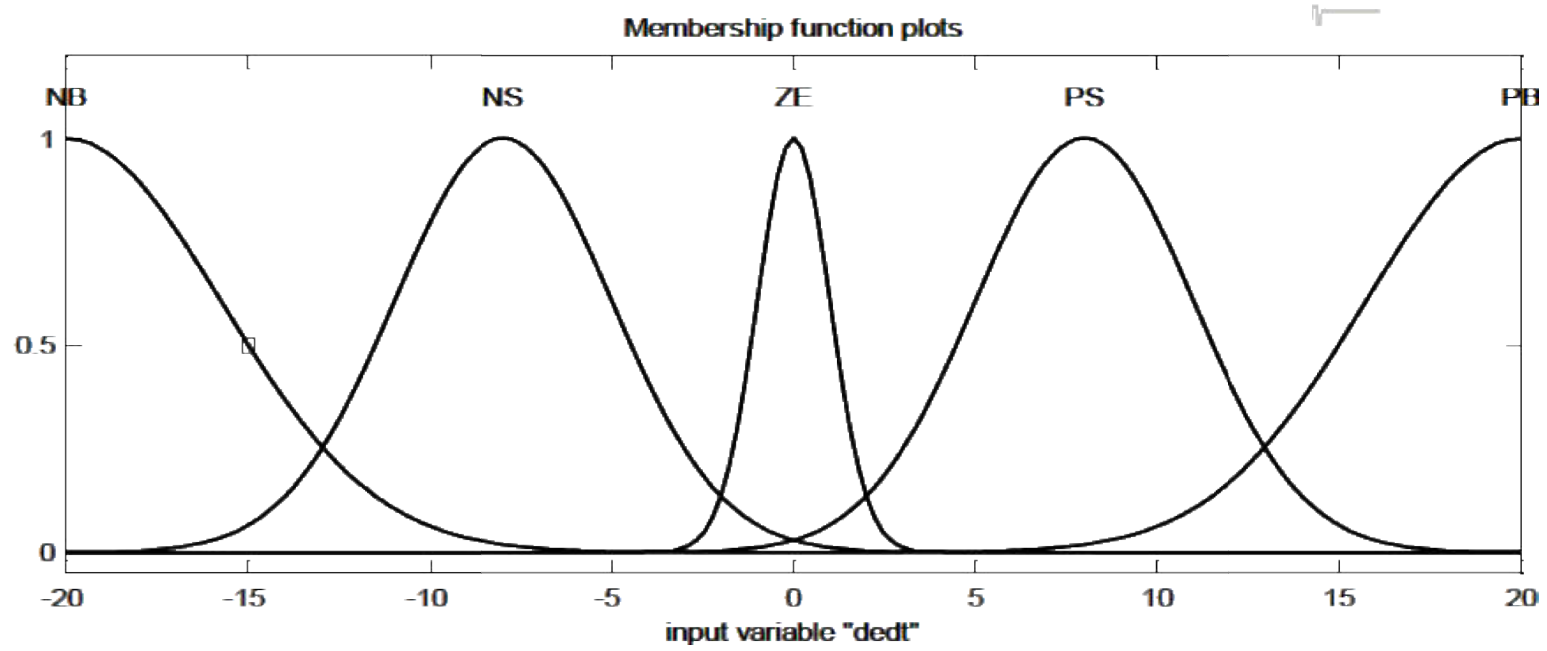

Fig.6. Membership function of rate of error

Membership functions for $K_{p}$ and $K_{i}$ were made common and normalized between -1 and 1 . They were divided into five regions proportionally and represented linguistically by Small (S), Medium Small (MS), Medium (M), Medium Big (MB) and Big (B). The representation of the membership function and its linguistic variables were described in Fig 7. 


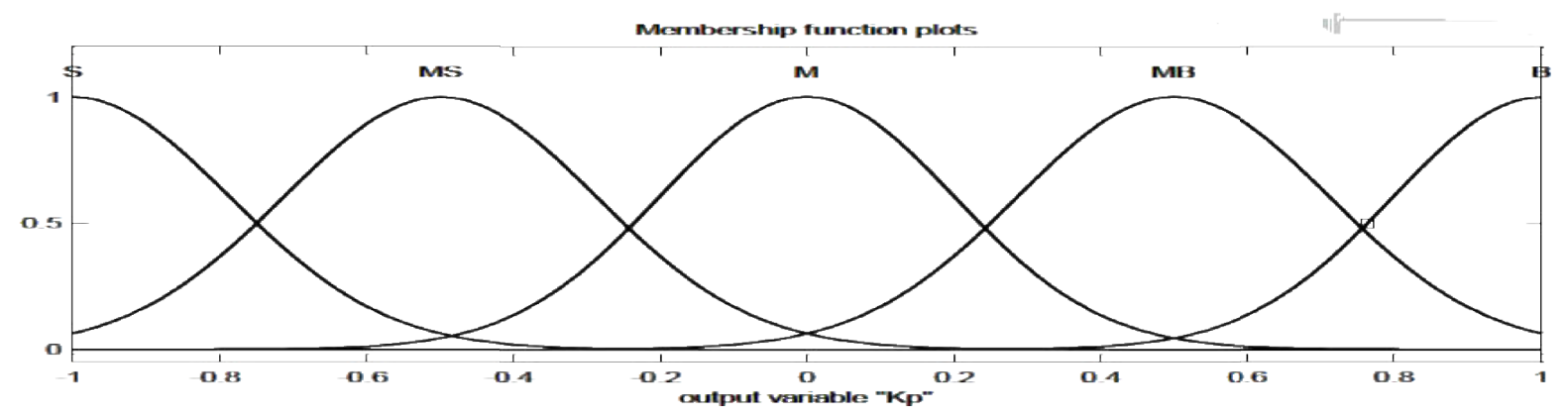

Fig.7. Membership function of PI gain

Range of each controller gain was set based on Ziegler-Nichols and AMIGO rules. Based on the rules, range of gain was set to, $1 \leq \mathrm{k}_{\mathrm{p}} \leq 6$ and $0.001 \leq \mathrm{k}_{\mathrm{i}} \leq 10.001$. The controller gain will be multiplied by the normalized gain determined from fuzzy. Fuzzy rules were constructed based on the information of error and rate of error to formulate the normalized controller gain. Each input was divided into five membership functions, and hence generated 25 fuzzy rules that relate to the output membership function. The fuzzy rules are listed in Table 1.

Table 1. Fuzzy rules

\begin{tabular}{cccccc}
\hline \multirow{2}{*}{ de/dt } & \multicolumn{5}{c}{ Error (e) } \\
& NB & NS & ZE & PS & PB \\
\hline NB & S & S & MS & MS & M \\
NS & S & MS & MS & M & MB \\
ZE & MS & MS & M & MB & MB \\
PS & MS & M & MB & MB & B \\
PB & M & MB & MB & B & B \\
\hline
\end{tabular}

\section{RESULTS AND DISCUSSION}

This section presents experimental results obtained from fuzzy PI, fuzzy FOPI and fuzzy FOPI with error filter used to regulate the steam temperature of the hydro-steam distillation plant. The set point was changed from $80^{\circ} \mathrm{C}$ to $85^{\circ} \mathrm{C}$ during each experiment to evaluate their performances. For fair comparison, every controller is set to the same membership functions and fuzzy rules. All controllers were evaluated based on their transient performances and integral of squared error (ISE) criteria. Other than that, to validate the objective of this research, quality analysis of essential oil extracted at $85^{\circ} \mathrm{C}$ and $100^{\circ} \mathrm{C}$ (uncontrolled) were also included.

\subsection{Controller Performance Evaluations}


Fig. 8 shows the output response of Fuzzy-PI and its control output during set point change. The controller produced large overshoot of $45.6 \%$ and the output was oscillating during steady-state. The steady-state error was estimated at $0.5{ }^{\circ} \mathrm{C}$. It was observed that the control output behaved like an ON/OFF controller that switched states when some error was detected. This behaviour was due to large gain settings in Fuzzy-PI controller.
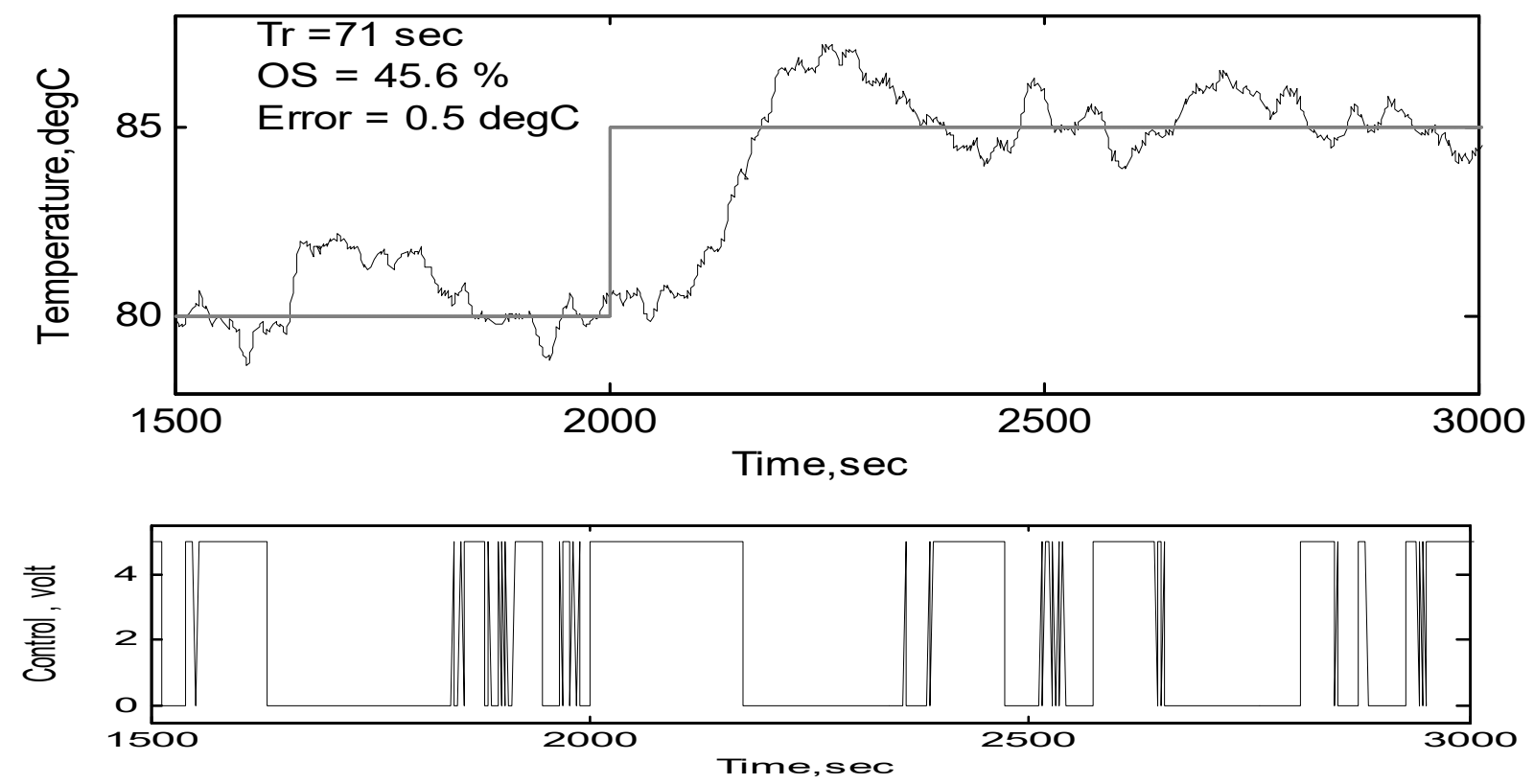

Fig 8. Fuzzy-PI response

However, the response was improved under Fuzzy-FOPI control as shown in Fig. 9. The overshoot was reduced compared to Fuzzy-PI but the output was still oscillating during steady-state. The steady-state error was estimated at $0.87^{\circ} \mathrm{C}$.
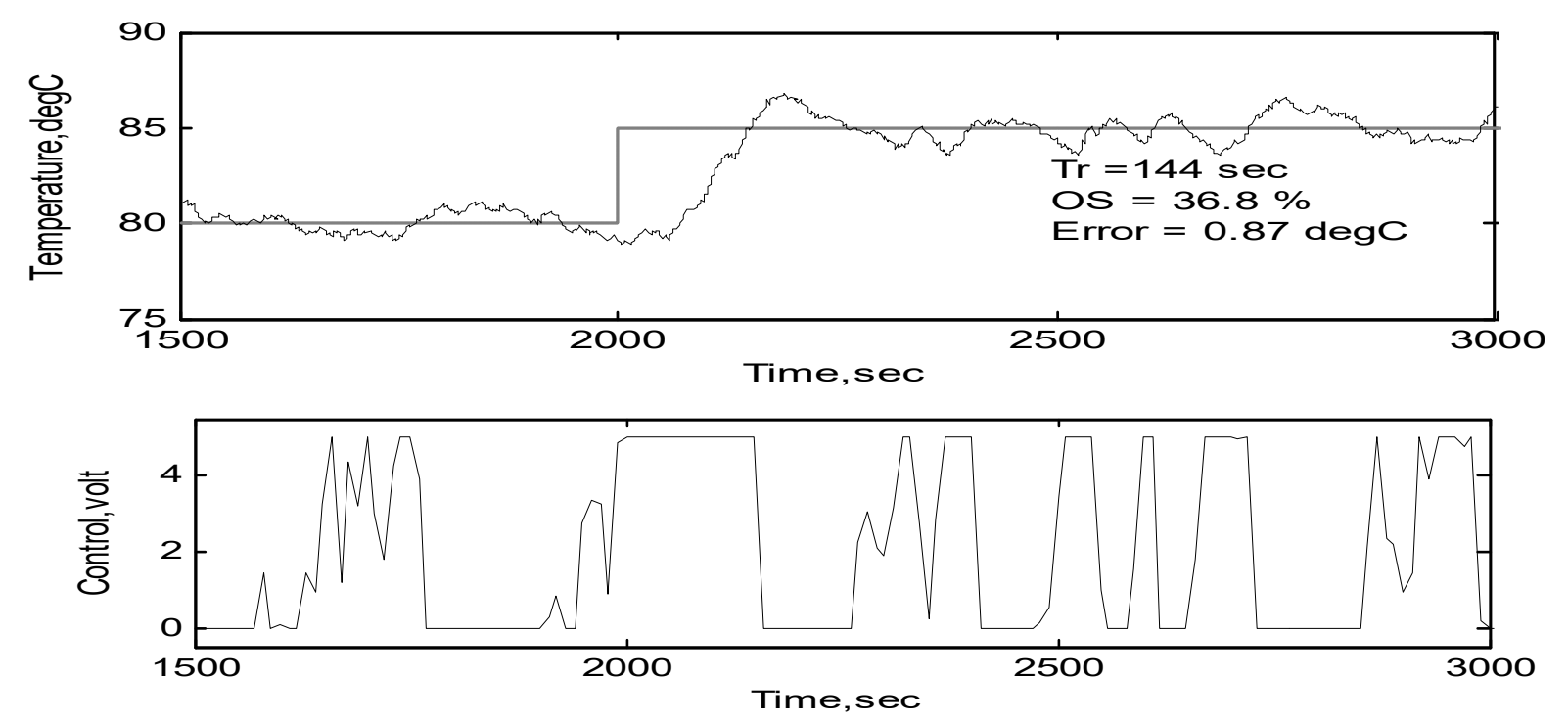

Fig.9. Fuzzy-FOPI response 
Steady-state error was improved by Fuzzy-FOPI with error filter as shown in Fig. 10. The overshoot was reduced to $22.2 \%$ and steady-state error was eliminated.
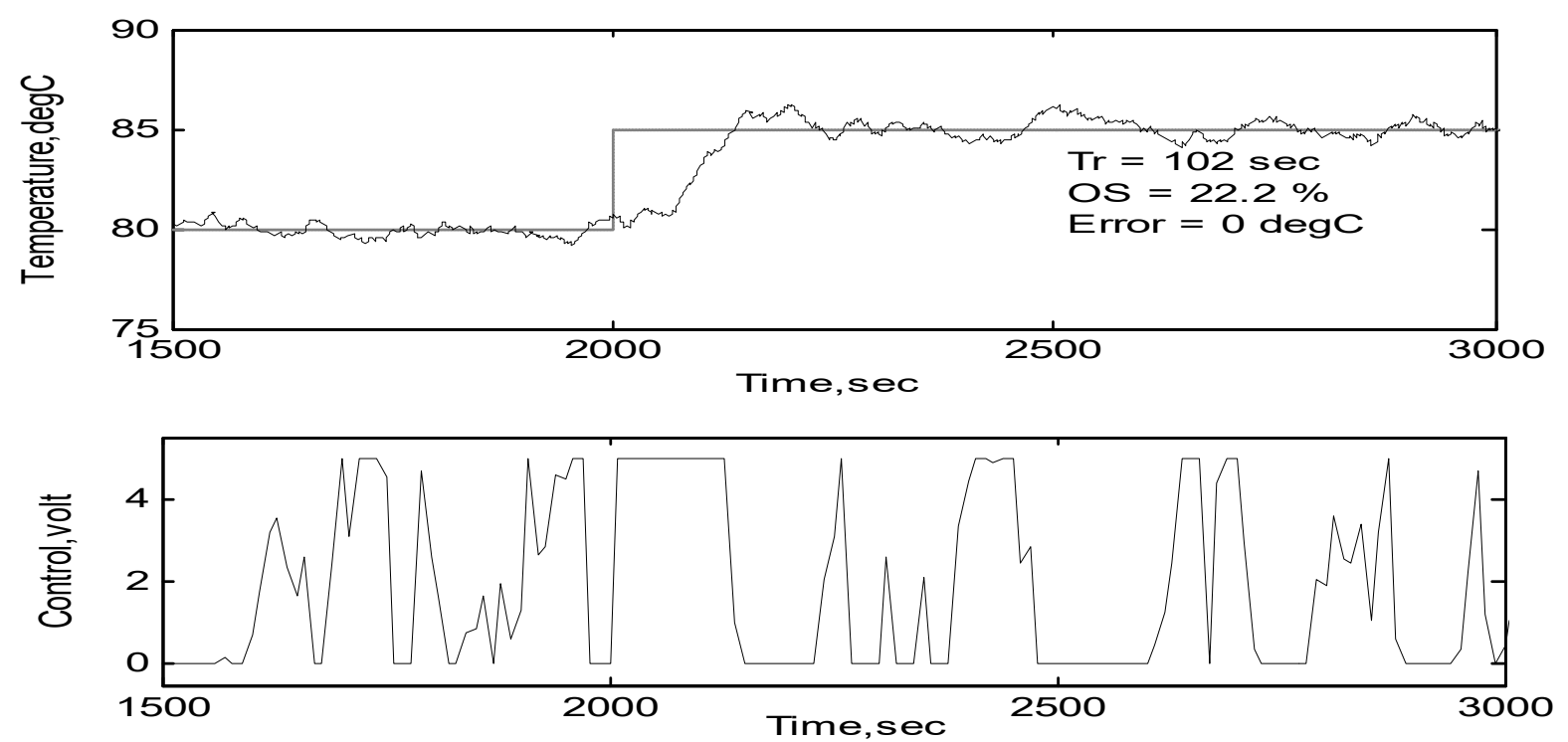

Fig.10. Fuzzy-FOPI with error filter Response

Output performances of the fuzzy controllers during set point change are summarized in Table 2. Fuzzy-FOPI had improved the overshoot of output response obtained from Fuzzy-PI to $36.8 \%$. However, Fuzzy-FOPI had increased the steady-state error from $0.5{ }^{\circ} \mathrm{C}$ to $0.87{ }^{\circ} \mathrm{C}$. Steady-state error was eliminated by Fuzzy-FOPIe control, which also produced the minimum overshoot of $22.2 \%$. Fuzzy-FOPIe also reduced the effect of oscillation during steady-state, but the output only settled after 563 seconds. Table 3 listed the error evaluation where fuzzy FOPI with error filter had minimum ISE when measured from $t=2000$ to 3000 seconds.

Table 2. Transient performance evaluations

\begin{tabular}{cccc}
\hline Controller & Rise Time (Sec) & Settling Time (Sec) & Overshoot (\%) \\
\hline Fuzzy-PI & 71 & Oscillating & 45.6 \\
Fuzzy-FOPI & 68 & Oscillating & 36.8 \\
Fuzzy-FOPIe & 102 & 563 & 22.2 \\
\hline
\end{tabular}

Table 3. Error performance criteria

\begin{tabular}{ccc}
\hline Controller & Steady-State Error $\left({ }^{\mathbf{0}} \mathbf{C}\right)$ & ISE $\left.(\mathbf{x 1 0})^{\mathbf{4}}\right)$ \\
\hline Fuzzy-PI & 0.5 & 0.398 \\
Fuzzy-FOPI & 0.87 & 0.373 \\
Fuzzy-FOPIe & 0 & 0.235 \\
\hline
\end{tabular}




\subsection{Essential Oil (C. nardus) Quality Assessment}

This section presents an analysis of citronella oil that was extracted at $85^{\circ} \mathrm{C}$ and $100^{\circ} \mathrm{C}$ using the proposed hydro-steam distillation system. The oil quality was assessed through its colour, refractive index and also the chemical composition from GC-MS analysis. Fig. 11 shows the appearance of citronella oil extracted at $85^{\circ} \mathrm{C}$ and $100^{\circ} \mathrm{C}$.

From the figure, it was observed that citronella oil extracted at $85^{\circ} \mathrm{C}$ appeared in pale yellow while the oil extracted at $100^{\circ} \mathrm{C}$ was more brownish. This physical appearance in the colour indicated the differences in composition between the two samples.

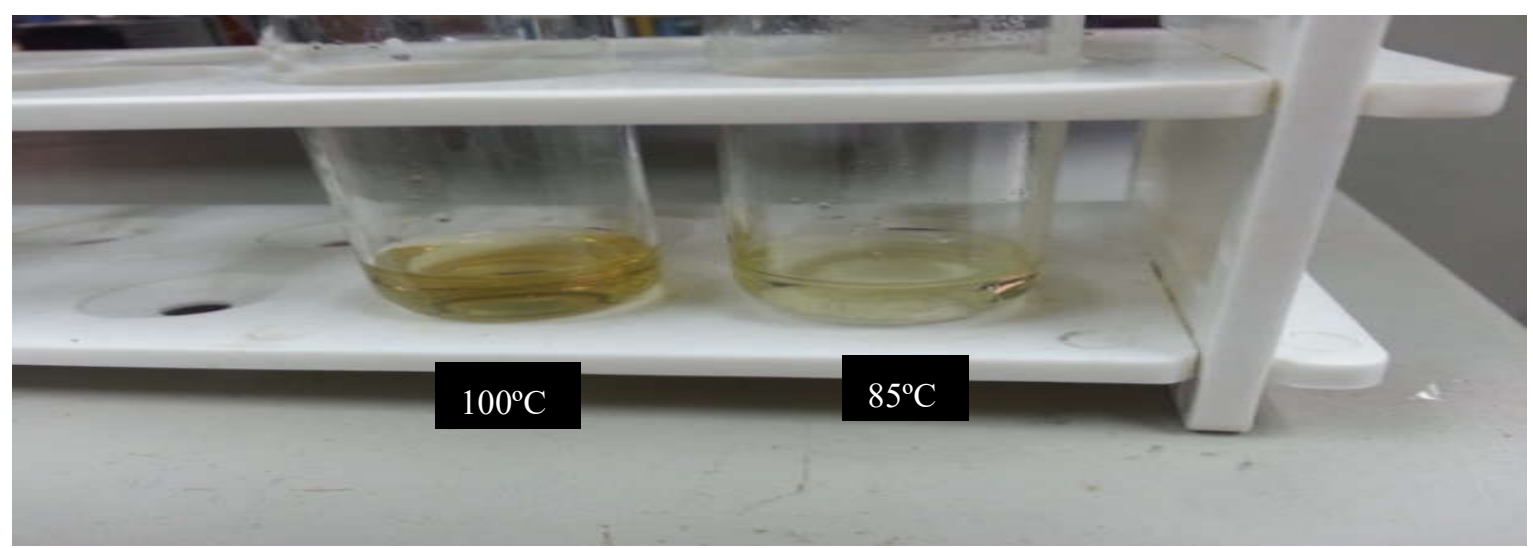

Fig.11. Physical appearance of citronella oil extracted at $85^{\circ} \mathrm{C}$ and $100^{\circ} \mathrm{C}$

Table 4. Refractive index of citronella oil extracted at $85^{\circ} \mathrm{C}$ and $100^{\circ} \mathrm{C}$

\section{Temperature $\left({ }^{\circ} \mathrm{C}\right) \quad$ Refractive Index}

\begin{tabular}{cc}
\hline 100 & 1.4747 \\
85 & 1.4710
\end{tabular}

Significant differences can also be observed from refractive index measurements given in Table 4. The index for citronella oil extracted at $85^{\circ} \mathrm{C}(1.4710)$ was lower compared to the oil extracted at $100^{\circ} \mathrm{C}(1.4747)$. This data also indicated the difference between citronella attribute that was extracted at different temperature, even though both data were lower than that specified by the BS ISO 3849:2003 which requires refractive index of 1.479 to 1.490 .

Table 5 lists the identified chemical compounds of citronella oil extracted at $85^{\circ} \mathrm{C}$ and $100^{\circ} \mathrm{C}$ obtained from the chromatograms. 
Table 5. Chemical compositions of citronella oil extracted at $85^{\circ} \mathrm{C}$ and $100^{\circ} \mathrm{C}$

\begin{tabular}{|c|c|c|c|}
\hline No. & Compound & $\begin{array}{c}85^{\circ} \mathrm{C} \\
\text { Area }(\%)\end{array}$ & $\begin{array}{c}100^{\circ} \mathrm{C} \\
\text { Area (\%) }\end{array}$ \\
\hline 1 & Limonene & 0.17 & 2.67 \\
\hline 2 & Linalool & 0.24 & 0.32 \\
\hline 3 & Citronellal* & 25.24 & 34.91 \\
\hline 4 & Citronellol* & 13.47 & 9.97 \\
\hline 5 & Geraniol* & 21.78 & 16.64 \\
\hline 6 & Citronellyl acetate & 2.60 & 1.99 \\
\hline 7 & Eugenol & 1.03 & 0.95 \\
\hline 8 & Geranyl butanoate & 3.17 & - \\
\hline 9 & Geranyl acetate & - & 2.49 \\
\hline 10 & $\beta$-elemene & 2.22 & 1.65 \\
\hline 11 & $\alpha$-humulene & 0.17 & - \\
\hline 12 & Germacrene D & 3.74 & 2.34 \\
\hline 13 & Germacrene A & - & 1.28 \\
\hline 14 & $\gamma$-cadinene & 0.37 & 0.24 \\
\hline 15 & Elemol & 5.73 & 8.47 \\
\hline 16 & Germacrene D-4-ol & 5.50 & 5.23 \\
\hline 17 & $\gamma$-eudesmol & 0.40 & 0.67 \\
\hline 18 & $\alpha$-cadinol & 1.57 & 1.57 \\
\hline & Total & 87.4 & 91.69 \\
\hline
\end{tabular}

Based on Table 5, sixteen compounds had been identified in citronella oil extracted at $100^{\circ} \mathrm{C}$ representing $91.69 \%$ of the detected compounds. The composition of major compounds included citronellal (34.91\%), citronellol (9.97\%) and geraniol (16.64\%). This composition was conformed to C. nardus extracts and was comparable to previous reports by $[1,23]$, but differed from [24-26]. However, the composition of the sample was much higher than the range specified by ISO 3849:2003 for citronellal (7.0\%-11.5\%), geraniol (3.0\%-8.5\%) and citronellol (4.0\%-7.0\%). 
Sixteen compounds had also been identified in citronella oil extracted at $85^{\circ} \mathrm{C}$ representing citronellal (25.24\%), citronellol (13.47\%) and geraniol (21.78\%) but total identified compounds was slightly lower which is $87.4 \%$. It was observed that both samples had the same major compounds but their compositions are varied with respect to extraction temperature.

\section{CONCLUSION}

This research had successfully implemented a real-time steam temperature control of a hydro-steam distillation plant for essential oil extraction process using self-tuning fuzzy fractional-order PI controller. The controller can regulate the steam temperature at $85^{\circ} \mathrm{C}$. The controller performances were significantly better when compared to self-tuning fuzzy PI and self-tuning fuzzy FOPI. This research also indicated positive improvements of citronella oil that was extracted at $85^{\circ} \mathrm{C}$ using the developed extraction system compared to the oil extracted at unregulated temperature.

\section{ACKNOWLEDGEMENTS}

This research is financially supported by UiTM internal fund Lestari (39/2015). The authors would like to thank the Faculty of Electrical Engineering, UiTM Selangor for facilities provided during this research. We also would like to express our gratitude to all members of PICon Research Interest Group for their comment and support.

\section{REFERENCES}

[1] Koba K, Sanda K, Guyon C, Raynaud C, Chaumont J P, Nicod L. In vitro cytotoxic activity of Cymbopogon citratus L. and Cymbopogon nardus L. essential oils from Togo. Bangladesh Journal of Pharmacology, 2008, 4(1):29-34

[2] Schaneberg B T, Khan I A. Comparison of extraction methods for marker compounds in the essential oil of lemon grass by GC. Journal of Agricultural and Food Chemistry, 2002, 50(6):1345-1349

[3] Nikolić N Č, Cakić S M, Novaković S M, Cvetković M D, Stanković M Z. Effect of extraction techniques on yield and composition of soybean oil. Macedonian Journal of Chemistry and Chemical Engineering, 2009, 28(2):173-179 
[4] Babu G K, Singh B I. Chapter 7-Characteristics variation of lavender oil produced by different hydrodistillation techniques. In V. K. Gupta, A. K. Verma, S. C. Taneja, \& B. D. Gupta (Eds.), Comprehensive bioactive natural products: Quality control and standardization. New Delhi: Studium Press LLC, 2010, pp. 122-136

[5] Mohamed N A. Study on important parameters affecting the hydrodistillation for ginger oil production. Master thesis, Johor: Universiti Teknologi Malaysia, 2005

[6] Hosni K, Kerkenni A, Medfei W, Ben Brahim N, Sebei H. Volatile oil constituents of Rosa canina L.: Quality as affected by the distillation method. Organic Chemistry International, 2010, 2010:1-7

[7] Galvão E L, Sant'Ana H B D, Oliveira H N M, Souza A V, Sousa E M B D. Influence of temperature in the kinetics extraction of the cymbopogon winterianus j. oil with dense carbon dioxide. In 2nd Mercosur Congress on Chemical Engineering and 4th Mercosur Congress on Process Systems Engineering, 2011, pp. 2-9

[8] Xue D, Chen Y. A comparative introduction of four fractional order controllers. In 4th IEEE World Congress on Intelligent Control and Automation, 2002, pp. 3228-3235

[9] Oustaloup A, Moreau X, Nouillant M. The CRONE suspension. Control Engineering Practice, 1996, 4(8):1101-1108

[10] Podlubny I. Fractional-order systems and PI $\lambda \mathrm{D} \mu$. IEEE Transactions on Automatic Control, 1999, 44(1):208214

[11] Wu Z, Li D, Wang L. Control of the superheated steam temperature: A comparison study between PID and fractional order PID controller. In 35th IEEE Chinese Control Conference, 2016, pp. 10521-10526

[12] Rastogi P, Chatterji S, Karanjkar D S. Performance analysis of fractional-order controller for $\mathrm{pH}$ neutralization process. In 2nd IEEE International Conference on Power, Control and Embedded Systems, 2012, pp. 1-6

[13] Merrikh-Bayat F. Rules for selecting the parameters of Oustaloup recursive approximation for the simulation of linear feedback systems containing PI $\lambda \mathrm{D} \mu$ controller. Communications in Nonlinear Science and Numerical Simulation, 2012, 17(4):1852-1861

[14] Feliu-Batlle V, Perez R R, Rodriguez L S. Fractional robust control of main irrigation 
canals with variable dynamic parameters. Control Engineering Practice, 2007, 15(6):673-686

[15] Budi L S, Wardhani R M. The application of fuzzy logic control systems on harvesting standard operating procedures models of sesame crops in Indonesia. International Journal on Advanced Science, Engineering and Information Technology, 2017, 7(3):1089-1097

[16] Mohd T A, Hassan M K, Aris I, Azura C S, Ibrahim B S. Application of fuzzy logic in multi-mode driving for a battery electric vehicle energy management. International Journal on Advanced Science, Engineering and Information Technology, 2017, 7(1):284-290

[17] Gupta S K, Varshney P. Fractional fuzzy PID controller for speed control of DC motor. In 3rd IEEE International Conference on Advances in Computing and Communications, 2013, pp. $1-4$

[18] Tian X, Huang Y, Zhang C. The tuning principle of adaptive fuzzy fractional-order PID controller parameters. Procedia Engineering, 2010, 7:251-255

[19] Sharma R, Rana K P, Kumar V. Performance analysis of fractional order fuzzy PID controllers applied to a robotic manipulator. Expert Systems with Applications, 2014, 41(9):4274-4289

[20] Mishra P, Kumar V, Rana K P. A fractional order fuzzy PID controller for binary distillation column control. Expert Systems with Applications, 2015, 42(22):8533-8549

[21] Liu L, Pan F, Xue D. Variable-order fuzzy fractional PID controller. ISA Transactions, $2015,55: 227-233$

[22] Tajjudin M, Ishak N, Rahiman M H, Adnan R. Design of fuzzy fractional-order PI+ PD controller. In IEEE 12th International Colloquium on Signal Processing and Its Applications, 2016, pp. 253-257

[23] Abena A A, Gbenou J D, Yayi E, Moudachirou M, Ongoka R, Ouamba J M, Silou T. Comparative chemical and analgesic properties of essential oils of Cymbopogon nardus (L) Rendle of Benin and Congo. African Journal of Traditional, Complementary and Alternative Medicines, 2007, 4(3):267-272

[24] Nakahara K, Alzoreky N S, Yoshihashi T, Nguyen H T, Trakoontivakorn G. Chemical composition and antifungal activity of essential oil from Cymbopogon nardus (citronella grass). Japan Agricultural Research Quarterly, 2013, 37(4):249-252 
[25] Setiawati W, Murtiningsih R, Hasyim A. Laboratory and field evaluation of essential oils from Cymbopogon nardus as oviposition deterrent and ovicidal activities against Helicoverpa armigera Hubner on chili pepper. Indonesian Journal of Agricultural Science, 2011, 12(1):9-16

[26] Chan L K, Dewi P R, Boey R L. Effect of plant growth regulators on regeneration of plantlets from bud cultures of Cymbopogon nardus L. and the detection of essential oils from the in vitro plantlets. Journal of Plant Biology, 2005, 48(1):142-146

[27] Nawi B, Sulaini B, Mohd Z A R, Shamsul A Z, Zairi I R. PID voltage control for DC motor using MATLAB Simulink and Arduino microcontroller. Journal of Applied Environmental and Biological Sciences, 2015, 5(9):166-173

[28] Masrom S, Abidin S Z Z, Omar N, Rahman A S A, Zairi I R. Dynamic parameterizations of particle swarm optimization and genetic algorithm for facility layout problem. ARPN Journal of Engineering and Applied Sciences, 2017, 12(10):3195-3201

\section{How to cite this article:}

Tajjudin M, Ishak N, Rahiman MHF, Adnan R. Steam temperature control of essential oil extraction system using fuzzy-fopi controller. J. Fundam. Appl. Sci., 2017, 9(4S), 238-255. 